\title{
Use of Free Open Source Software Technologies to Enhance Knowledge Mobilization in Smallholder Agricultural Communities in Sri Lanka
}

\author{
H.A.C.K. Jayathilake*, U.K. Jayasinghe-Mudalige ${ }^{1}$, L.D.R.D. Perera ${ }^{2}$ \\ G.A. Gow ${ }^{3}$ and N. Waidyanatha ${ }^{4}$ \\ Information and Communication Technology Centre \\ Wayamba University of Sri Lanka \\ Sri Lanka
}

\begin{abstract}
Development of credible approaches to share indigenous and scientific knowledge in possession of farmers, especially those engage in rural subsistence agriculture, has become a necessity in order to enhance their competitive edge in agriculture, however, remains as a challenge at present. The concept of Knowledge Mobilization (KMb) has, in the light of this, gained much prominence, where the role of information and communication technologies to promote sustainable agriculture is of greater attention. On this rationale, this study was carried out to explore the possibility of applying Free Open Source Software (FOSS) technologies, in combination of mobile technologies, to promote sustainable knowledge mobilization amongst the agricultural communities in Sri Lanka. Multi-stage community consultations with designated officials (partners) and pre-tested structured questionnaire-based face-to-face interviews with randomly selected smallholder agriculture farmers (n=272) from Batticaloa, Kurunegala, Matale and Puttalam Districts were employed to gather baseline data. Eight campaigns were carried out with the farmers ( $n=720)$ from the same geographical areas, where the three FOSS technologies, namely: (1) FrontlineSMS for Texting (2) FreedomFone for Interactive Voice Responses or Voice-Call and (3) Ushahidi for Interactive mapping, were applied to evaluate the possibility and effectiveness of knowledge sharing within those farming communities. It was revealed that FOSS intervention augments the Texting, Voice-Call and Interactive-mapping usage in dayto-day agricultural communication by 21,18 and 5 percent, respectively. The key demographic factors considered, including the age and educational levels of farmers have triggered the process of knowledge mobilization positively. Outcome of the study, overall, infers that, with a fitting mechanism in place, this approach can be promoted as a drive for positive changes in agriculture-based rural communities in developing countries like Sri Lanka.
\end{abstract}

Keywords: Agricultural community, FOSS, information, knowledge mobilization

\footnotetext{
Department of Agribusiness Management, Wayamba University of Sri Lanka, Sri Lanka

Department of Computing and Information Systems, Wayamba University of Sri Lanka, Sri Lanka

Faculty of Extension, University of Alberta, Canada

4 LIRNEasia, Sri Lanka

* Corresponding author : hackjayathilake@gmail.com
} 


\section{INTRODUCTION}

Knowledge plays an indispensable role in the process of transforming the livelihoods of farming communities, especially those who relies on subsistence agriculture in developing countries. In many developing countries the majority of the population often depends on agriculture for their livelihoods but the yields from agriculture are very low when comparing to developed countries. A major cause for this is the lack of information and knowledge about farming for smallholder farmers (FAO, 2015). Further constraints and limitations on traditional agricultural extension methods as well as high costs of information provision have been cited as barriers to improving the livelihood of farmers in developing countries like Sri Lanka and belong to the subsistence agricultural systems (Rivera et al., 2009; De Silva et al., 2011). Thus, the development of credible approaches to share the indigenous and scientific knowledge in possession of farmers in order to enhance their competitive edge in agriculture has become a necessity at present, but a challenge.

Knowledge mobilization refers to moving or sharing available knowledge (often from formal research) into active use and this involves knowledge sharing between research producers (e.g. university / research institute) and research users (including professionals or others whose work can benefit from research findings) often with the help of third parties or intermediaries (Gow et al., 2013; Furgal, 2014). The knowledge mobilization in support of sustainable agriculture has been identified as a vital activity that faces numerous challenges today (Aker, 2010). It is argued that the use of Information Communication Technologies (ICTs) can play a key role in sharing knowledge, connecting farmers to information to enable more effective resource use, stimulate agricultural innovation and create resiliency within agri- food systems (De Silva et al., 2011; World Bank, 2011). Incorporation of ICTs into agricultural systems could, therefore, be taken as an effective solution to overcome such issues. Many authors (Shove et al., 2012; Gow et al., 2013) pointed out that low cost FOSS platforms can be used in knowledge sharing approaches especially in participatory design processes in rural communities. The adoption and use of digital information services as such is, however, closely related to how well these services are aligned with the everyday social practices of people (De Silva et al., 2011). Thus, this is suggested that an existence of such services satisfies a 'necessary' condition, but is not 'sufficient' to foster the development and deployment of new services effectively.

In Sri Lankan context, plenty of agricultural knowledge has been generated through various domains (e.g. Universities, Research Stations and Government Departments) in different forms. For example day-to-day communications on prevailing "market prices" and "weather conditions" to reviews and reports that are released periodically. Nevertheless, the smallholder agricultural communities in the country, especially in less-developed rural areas, have persistently suffered by issues related to receipt of appropriate knowledge and information pertaining to agriculture on time. This has mostly been accumulated to the inefficiencies associated with the mode of dissemination (De Silva et al., 2011; Jayathilake et al., 2015).

And also, the agricultural technologies and developments should reach farmers in its best form and through the most economical route (right information to right people at right time), otherwise transforming sustainable agriculture into productive systems would become an unrealizable effort. Therefore, the relevant agricultural knowledge repositories should come up with new technologies and developments and further attention must be given to expose them to the potential farmers. This study examined the possibility of applying three FOSS namely FrontlineSMS (FLSMS), FreedomFone (FF) and Ushahidi to promote sustainable 
knowledge sharing in ICT-enable farming communities in Sri Lanka. Shove et al., (2012) and Gow et al., (2013) point out the significance of incorporating this concept with the ICTs to share the knowledge effectively.

'FrontlineSMS' is a FOSS which enables users to connect a range of mobile devices to a computer to send and receive Short Message Service (SMS) communication. The software works without an internet connection by connecting a device such as a cell phone or GSM (Global System for Mobile communications) modem plugged into the computer via the USB drive with a local phone number. FLSMS can be used to send and receive messages, group contacts, respond to messages, and trigger other events. If internet access is available, FLSMS can be connected to online SMS services and set up to feed incoming messages to other web or e-mail services (Frontline SMS, 2013).

'Freedom Fone' is FOSS, enables users to create and share audio content using IVR (Interactive Voice Responses), voicemail and SMS. FF is used to create two-way phonebased communication services to interact with any audience, in any language, at any time and without recourse to internet or other media. Without any geographical limitations, FF can be used in any country with mobile network coverage and runs on Linux distributions that are based on Ubuntu, Debian Linux operating systems especially on Debian and Ubuntu. (Clark and Burrell, 2009).

'Ushahidi' is a FOSS built on the Kohana web framework, a fork of the Code Igniter framework for information collection, visualization and interactive mapping. 'Ushahidi' provides the option of using Open Street Map maps in its user interface, but requires the Google Maps API (Application Program Interface) for geocoding. This platform now has allowed anyone around the world to set up their own way to gather reports in an incidencereporting and mapping by mobile phone, email and the web and map them (Donner et al., 2008).

\section{METHODOLOGY}

The 'Sponsors' for this multi-phased research study were identified through a national workshop on 'Agriculture Knowledge Mobilization' conducted by the Wayamba University of Sri Lanka in collaboration with University of Alberta and LIRN Easia. Four institutions were selected as the Sponsors namely the "Janathakshan" (non-governmental organization) in Batticaloa, Department of Export Agriculture (DOEA) in Kurunegala, Rangiri radio in Matale and Ag-Voice Federation (AVF) in Puttalam, which are working closely with the field level.

Series of discussions and meetings were held with communities from Janathakshan, DOEA, Rangiri and AVF sponsors. During each of these meetings, the research team was able to identify a major problem from its community and in turn, immediately furnish quick solutions by configuring FOSS (FLSMS/FF/Ushahidi) and testing with the community, called a 'rapid prototyping' exercise. This rapid prototyping practice was a process of trial and error with quick turnaround times with FOSS used. Testing and refining a technology platform quickly helps to keep up interest and momentum, reduces costs, and provides immediate feedback on the design of the system in order to improve it for users. FLSMS and FF were used as its starting point. This envisaged the community's knowledge and hands on experience of using these FOSS applications activities. 
A face-to-face interview supported by a pre-tested structured questionnaire was conducted with a proportionate randomly selected sample of 272 farmers from the Janathakshan $(\mathrm{N}=98)$, DOEA $(\mathrm{N}=85)$, Rangiri $(\mathrm{N}=26)$ and AVF $(\mathrm{N}=63)$. The 'LIRNEasia Teleuse@BOP4' (LIRNEasia, 2012) instrument was adopted, in particular, for the purpose of preparation of questionnaire to gather information pertaining to the socio-economic and demographic data of respondents as well as their behavior on agriculture information seeking. The survey provided insights as to how, when, and why ICTs were used in their daily lives, for example. Wenger et al. (2009) showed that the spider diagram can be used to create an orientation profile for a community. This was a very helpful tool to organize our community activities and led to select technology tools. Thus, next, 'spider diagram' tool was used to create an orientation profile for a community. Based on the information gathered from each community, a detailed list of requirements from the user's point of view were identified and created the relevant 'Use Case Diagrams' and designed respective 'campaign'. A 'campaign' is an activity that runs for a certain period of time (i.e. 3 to 4 months) with a specific objective to reflect the information sharing or need of the respective community. Each community ran two parallel campaigns comprising 650 smallholder agricultural farmers.

After the campaign, another series of face-to-face interviews, supported by previous semistructured questionnaire were carried out with the same participants $(\mathrm{n}=272)$, and the "pre" and "post" campaign survey data and FOSS technology usage data from the 'messaging', 'IVR' and 'mapping' software deployed in the campaigns were analyzed. The amount of agriculture related SMS transferred, voice message recorded and reports generated from different geo-locations were used for measuring knowledge mobilization. The whole process was carried out during April 2014 to October 2015 for about 19 months uninterruptedly.

The nature of data on agriculture information need is dichotomous. The Probit model was applied in particular to assess whether farmers' agricultural information need (through ICTs) is correlated with demographic factors like their level of age, education and household income. Campaigns were conducted in two time slots in each community groups and the farmers' SMS use, IVR use and Mapping for knowledge mobilization were measured before and after the campaign. Therefore, this represents a panel data set compiled in two time periods. The GEE (Generalized Estimating Equations) approach was developed by Zeger and Liang (1986) to produce more efficient and unbiased regression estimates for use in analyzing longitudinal or repeated measures research designs with non-normal response variables. The generalized linear model fits and allows specifying the within-group correlation structure for the panel data. In Stata, xtgee approach was used to analyze the campaign effect and the SMS, IVR and Mapping usage.

\section{RESULTS AND DISCUSSION}

The findings from the present study showed that the majority of the respondents in study communities were males (72\%). Mean age of the respondents was 48 years with a standard deviation of 13.8. It can be observed that more than 50 percent of the farming communities in the study area are middle aged and thus can be assumed as experienced farmers. As a whole, these farmers are in their middle ages that help grasping those information and communication technologies speedily and effectively. These findings agree with that of Lwoga, who argues that middle age farmers acquire ICTs effectively (Lwoga, 2011). 
When considering the ethnicity of the communities under investigation, the majority of farmers participated were 'Sinhalese' (62\%). There were 35 percent of Tamils and 3 percent of Muslims. The distribution of ethnicity was connected to the geographical location. During the focus group discussions it was found that knowledge mobilization pattern was slightly different among the different ethnic groups aggregated geographically. These findings are in line with the findings of Röling (1989) who claimed that ethnicity, population density and infrastructure and diversity in agro-ecology influence local agricultural knowledge.

Considering the annual income level, majority (60 percent) of the farmers were in lowest income category (less than LKR: 180,000) and 30 percent of the farmers were in middle income category (between LKR: $180,000-360,000)$ and rest $(10$ percent) were in the highest income category (higher than LKR: 360,000). The average annual income of the farmers in the studied community was reported as LKR: 157,064.00. Majority of the lowest income category farmers are doing subsistence agriculture and the surplus is sold. Probably, less than $50 \%$ of the crops they grow are used to generate income for their family. And also their cultivating land extent is limited. Therefore, annual household income of the majority of the small holder agricultural farmers was less than LKR: 180,000/=.

By considering the education level, most (97 percent) of the respondents had some level of formal schooling and this highlighted that majority could also read and understand simple instructions. However, about $80 \%$ of them have not received education beyond Grade 11 and consequently their competency in ICT could be low. There were about $3 \%$ of farmers who were illiterate. This situation should be taken up during the designing and implementation of campaigns about ICT tools for smallholder farmer communities. Very few farmers in the communities under investigation $(<1 \%)$ have had post-secondary educational qualifications. This situation is a common feature among smallholder farmers in developing countries which has been confirmed by many studies done in the developing world (Kaniki, 2004).

Level of information need of persons or groups of persons may be variable depending on a variety of factors, such as demographic factors (education, age etc.), social factors, economic background and geographic location (Kaniki, 2001). This was further tested and understood in the Sri Lankan context by fitting a linear model to binary response on necessity of information by farmers. Out of the different models; logit, probit, complementary log-log fitted to data, the probit model was found to be the most promising model (AIC $=107.45$ ) of which the results are given in Table 1.

Table 1. Outcome of the Probit Model - information need

\begin{tabular}{lcc}
\hline Variables & \multicolumn{2}{c}{ Probit } \\
\cline { 2 - 3 } & \multicolumn{1}{c}{ coefficient } & $\mathbf{d y} / \mathbf{d x}$ \\
\hline Gender (sex) & $0.3596^{+}(0.252)$ & 0.0418 \\
Age (age) & $-0.0370^{*}(0.009)$ & $-0.0043^{*}$ \\
Education level (edu) & $0.1256^{*}(0.048)$ & $0.0146^{*}$ \\
Household income (inc) & $0.2378(0.288)$ & 0.0276 \\
Location (com) & $-0.0206(0.073)$ & -0.0024 \\
\hline - Marginally significant (at 0.1 level), *- significant at 0.05 level & \\
Values within parentheses are robust standard errors &
\end{tabular}


It can be found that age and education are statistically significant at $5 \%$ level while the gender is marginally statistically significant (10\% level) on information need by the farmers in a community. Consequently, it can be inferred that most critical factors that decide information need by the subsistent farmers in the communities under the investigation are their gender, age and level of education. These findings agree with that of Lwoga, who argues that age and gender have impact on knowledge seeking (Lwoga, 2011). The covariate "community" found to be statistically insignificant which indicates that information need by subsistent farmers is indifferent over different communities. This information can be further utilized for planning various activities including policy formulation relevant to subsistent farming communities.

It can be observed that of the subsistent farmers, the interest of seeking knowledge (information need) decrease with the increase of their age. Older farmers in these communities pay less attention to get agricultural information compared to that of the young farmers which confirm the fact that older farmers (Digital immigrants) are less quick to pick up new technologies than young farmers (digital natives). Adeogun et al. (2010) had also orated that; the younger farmers would mostly be willing to spend more time to obtain information on improved technologies compared to the older farmers.

Majority of farmers in these areas cultivated vegetables and export agricultural crops such as ginger, pepper and cinnamon. Additionally, they grew rice and maize. 'Mobile Telephony' has been rated as the most extensive mode of communication within these communities in their day-to-day agricultural communication.

During field testing of the FOSS implementation, respondents were asked to indicate the use of SMS and IVR facility in their daily lives for communication related to agricultural activities "after" the campaigns. The respondents were asked whether they have used SMS and IVR or not and how many times they have been used. This information was gathered twice; before and after the campaign. Therefore, data are dichotomous in nature and constitute as a panel and thus the xtgee approach in STATA Version 14.0 was used to analyze the campaign effect where a Generalized Estimating Equation (GEE) is estimated. This enables the extraction of true effect of the treatment (campaign) on the use of SMS or IVR, from that of natural increase of use of SMS/ IVR over time. The results are depicted in Table 2.

Table 2. Outcome of the GEE Model - SMS and IVR usage in all communities

\begin{tabular}{llllll}
\hline Variable & & SMS use & IVR use & \\
\cline { 3 - 5 } & & Coefficient & Std. Err & Coefficient & Std. Err \\
\hline Gender $($ sex $)$ & & 0.197 & 0.214 & -0.012 & 0.406 \\
Age & (d_age $)$ & $-0.879^{*}$ & 0.573 & $-0.460^{*}$ & 0.581 \\
& (d_age3) & -0.891 & 0.631 & -0.139 & 0.588 \\
& (d_age4) & -1.367 & 0.662 & 0.163 & 0.648 \\
& (d_age5) & $-1.448^{*}$ & 0.743 & - & - \\
Education & (d_edu2) & -0.340 & 0.580 & 2.348 & 2.652 \\
& (d_edu3) & 0.712 & 0.558 & 1.516 & 2.623 \\
& (d_edu4) & 1.109 & 0.694 & 2.356 & 2.597 \\
Income & (d_inc1) & -2.146 & 0.684 & -0.801 & 0.560 \\
\hline
\end{tabular}




\begin{tabular}{lllll}
\hline \multicolumn{1}{c}{$\left(d \_\right.$inc 2$)$} & -1.461 & 0.704 & -0.220 & 0.404 \\
Before Campaign & 0.390 & 0.266 & 1.523 & 0.930 \\
After Campaign & $0.870^{*}$ & 0.260 & $2.144^{*}$ & 0.940 \\
\hline$*$ - significant at 0.05 level & &
\end{tabular}

By comparing the respondents' SMS and IVR usage before and after campaigns in all communities, Table 2 results show that the SMS and IVR usage has significantly increased after the campaign. With this result it can be inferred that knowledge mobilization of a community can be effectively improved by employing a SMS and/or IVR campaign. In the meantime, other covariate age showed a statistically significant impact on their knowledge mobilization. Consequently, the best way to improve the agricultural KMb with the aid of FOSS tools (FLSMS or FF) is to have low-cost software implementation.

A Comparison of ICT devices and services usage in the entire study from baseline survey to post campaign survey is depicted in Figure 1.

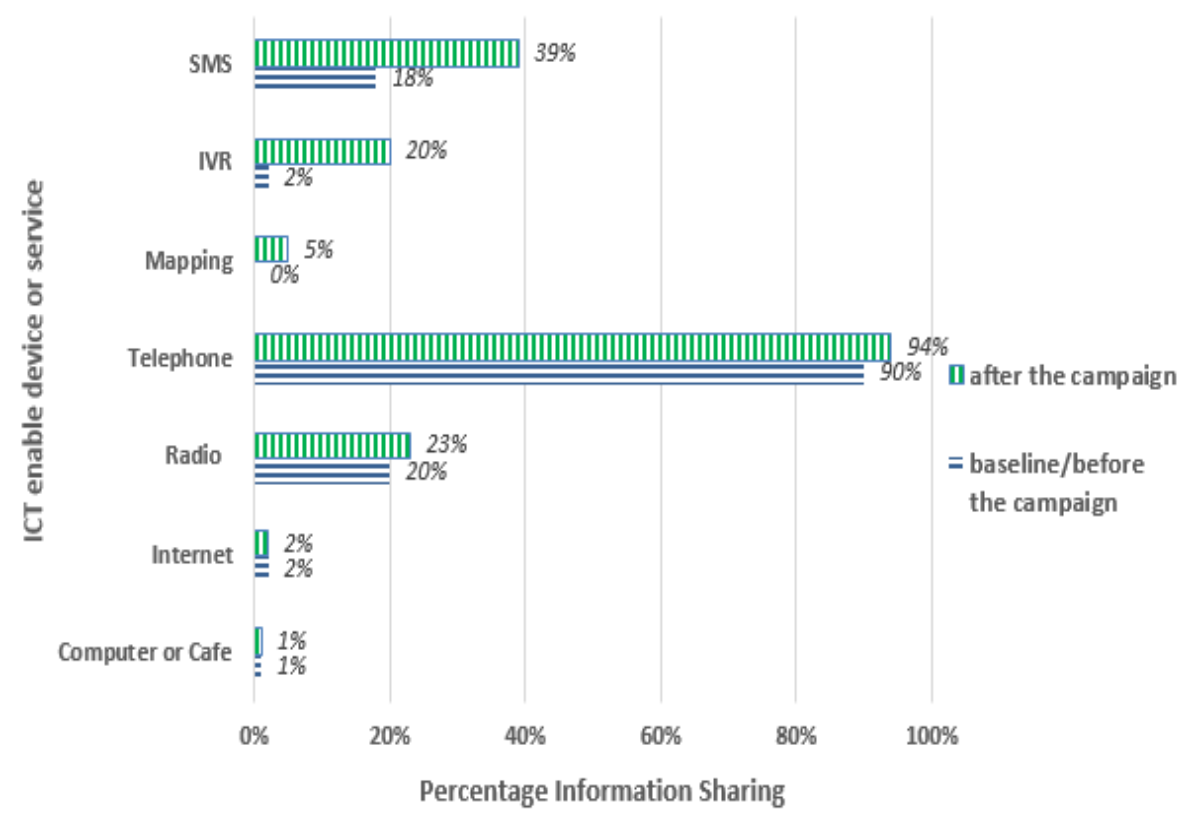

Figure 1. Comparison of the ICTs use in knowledge sharing before and after campaign

A significant change in the use of various devices for communication cannot be observed before and after the campaigns. The community use the telephone most frequently compared to the use of other devices such as radio, television and computer which enable to implement ICT solutions by using the telephones among these communities. Use of computer by these communities remained at minimum level. However, it can be further observed from these results that average SMS usage has increased by 21 percent, mapping usage has increased by 5 percent while the average usage of IVR has augmented by 18 percent after the campaigns. These results (SMS and IVR) are in line with the results shown by the model fitting exercise in Table 2. 
It was identified that this FOSS deployment was quite successful as the farmers got the cheap reliable agricultural information of the project. This intervention can be promoted, with necessary modifications like capacity development and introduction of comprehensive training modules for further learning, to work as a drive for positive changes in the livelihood of agriculture-based rural communities. In capacity building process developing and delivering a course curriculum that addresses both technical and community engagement aspects was identified as very important for local innovation with low cost ICTs. Once developed, this approach can play an imperative role in advancing knowledge sharing, starting from the lowest socio-economic category in farming communities.

\section{CONCLUSIONS}

It was found that FOSS intervention increases the 'Texting', 'Voice-Call' and 'Interactivemap' usage in day-to-day agricultural communication. The demographic factors like age and educational level of the farmers have positively influenced on the knowledge mobilization process. And also the 'Mobile Telephony' was the most extensive mode of communication within the communities.

The outcome of this multi-phased research study highlights that the possibility of applying FOSS technologies to promote sustainable knowledge mobilization in the said districts plays a prominent role to change the way farmers communicate and get solutions to their burning issues in agriculture. This FOSS deployment can be usefully conceived of as a micro-level innovation system. In fact, once developed, this approach can play an imperative role in advancing knowledge mobilization, starting from the lowest socio-economic category in farming communities. The stakeholders work on these criteria shall be regulated by relevant line Ministries working for agriculture to make sure 'right information is shared' and facilitated with various market-based incentives to 'augment the level of usage'. Future research is necessary to design more formal training programs for ICTs familiarization with converging FOSS that addresses both technical and community engagement aspects with a view to building community capacity for local innovation with low cost ICTs.

\section{ACKNOWLEDGEMENT}

The authors express their appreciation to the Ag-Voice Federation, Department of Export Agriculture, Rangiri and Janathakshan for their invaluable contributions made and their commitment to this project.

\section{REFERENCES}

Adeogun, S.O., Olawoye, J. E. and Akinbile, L.A. (2010). Information sources to cocoa farmers on cocoa rehabilitation techniques (CRTs) in selected states of Nigeria. J. Media Commun. Stud. 2(1), 9.

Aker, J. (2010). Information from markets near and far: Mobile phones and agricultural markets in Niger. Am. Econ. J. Appl. Econ. 2(3), 46-59. 
Clark, B. and Burrell, B. (2009). Freedom fone: dial-up information service. pp. 483-483. In: Information and Communication Technologies and Development (ICTD), International Conference on IEEE.

De Silva, H., Ratnadiwakara, D. and Zainudeen, A. (2011). Social influence in mobile phone adoption: Evidence from the bottom of pyramid in emerging Asia. Information Technologies and International Development. 7(3), 1-18.

Donner, J., Verclas, K. and Toyama, K. (2008). Reflections on MobileActive08 and the M4D Landscape. pp. 73-83. Proceedings of the First International Conference on M4D.

FAO (2015). The State of: Social protection and agriculture: breaking the cycle of rural poverty. Food and Agriculture Organization, United Nations.

FrontlineSMS. (2013). [Accessed on 21.04.2015]. Available at http://en.wikipedia.org/wiki/FrontlineSMS

Furgal, C. (2014). Knowledge mobilization: From knowledge to action [on line]. [Accessed on 25.04.2015]. Available at http://heicresearch.com/?p=1514

Gow, G., Hambly, H., Jayathilake, C. and Waidyanatha N. (2013). Final report: 2013

workshop on supporting sustainable agricultural communities of practice with low cost ICTs, [Online]. [Accessed on 25.04.2015]; Available at

https://docs.google.com/a/ualberta.ca/file/d/0BwjZ8EhyDFTNSW5xck1BUUhRTjQ/edit?us $\mathrm{p}=$ sharing

Jayathilake, H.A.C.K., Jayasinghe-Mudalige, U.K., Gow, G.A., Waidyanatha, N. and Perera, L.D.R.D. (2015). Use of low cost information communication technologies for knowledge mobilization in agricultural communities in Sri Lanka. $8^{\text {th }}$ International Research Conference.

Kaniki, A. (2004). Information needs for basic research: an African perspective. (p. 81). In: open access and the public domain in digital data and information for science: Proceedings of an International Symposium. National Academies Press, Washington, United States.

Kaniki, A.M. (2001). Community profiling and needs assessment. Knowledge, information and development: an African perspective. Scottsville, South Africa: School of Human and Social Studies, University of Natal (Pietermaritzburg). pp. 187-199.

LIRNEasia (2012). Teleuse at the Bottom of the Pyramid 4 (Teleuse@BOP4) [online]. [Accessed on 22.04.2017]. Available at http://lirneasia.net/projects/2010-12-researchprogram.

Lwoga, E.T. (2011). Knowledge management approaches in managing agricultural indigenous and exogenous knowledge in Tanzania. J. Doc. 67(3), 407-430.

Rivera, W.M. and Rasheed S.V. (2009). Extension: object of reform, engine for innovation. Outlook on Agriculture, 38(3), 267-273.

Röling, N.G. (1989). The research/extension interfaces a knowledge system perspective. International Service for National Agricultural Research Staff Notes. pp. 89-48. 
Shove E., Pantzar, M. and Watson, M. (2012). The dynamics of social practice: Everyday life and how it changes. Sage, Newcastle upon Tyne, United Kingdom.

Wenger, E., White, N. and Smith, J.D. (2009). Digital habitats: Stewarding technology for communities. CPsquare, Portland.

World Bank (2011). E-sourcebook: ICT in Agriculture: Connecting smallholders to knowledge, networks, and institutions. The International Bank for Reconstruction and Development / The World Bank, Washington, DC, USA.

Zeger, S.L. and Liang, K.Y. (1986). Longitudinal data analysis for discrete and continuous outcomes. Biometrics. pp. 121-130. 